\title{
Adnexal Carcinoma
}

National Cancer Institute

\section{Source}

National Cancer Institute. Adnexal Carcinoma. NCI Thesaurus. Code C3775.

A carcinoma arising from the sebaceous glands, sweat glands, or the hair follicles.

Representative examples include sebaceous carcinoma, apocrine carcinoma, eccrine carcinoma, and pilomatrical carcinoma. 